\title{
Robust circular distance and its application in the identification of outliers in the simple circular regression model
}

\begin{abstract}
Abstract: Background and Objective: The existence of outliers in any type of data influences the efficiency of an estimator. Few methods for detecting outliers in a simple circular regression model have been proposed in the study but it suspected that they are not very successful in the presence of multiple outliers in a data set. This study aimed to investigate new statistic to identify multiple outliers in the response variable in a simple circular regression model. Materials and Methods: The proposed statistic is based on calculating robust circular distance between circular residuals and circular location parameter. The performance of the proposed statistic is evaluated by the proportion of detected outliers and the rate of masking and swamping. The simulation study is applied for different sample sizes at 10 and $20 \%$ ratios of contamination. Results: The results from simulated data showed that the proposed statistic has the highest proportion of outliers and the lowest rate of masking comparing with some existing methods. Conclusion: The proposed statistic is very successful in detecting outliers with negligible amount of masking and swamping rates.
\end{abstract}

Keyword: Circular regression; Outliers; Masking; Swamping 